\title{
ESTUDO COMPARATIVO DA ESTIMATIVA DA EVAPOTRANSPIRAÇÃO DE REFERÊNCIA PARA TRES LOCALIDADES DO ESTADO DO ESPÍRITO SANTO NO PERIODO SECO
}

\author{
COMPARATIVE STUDY OF THE ESTIMATE OF THE EVAPORATE \\ TRANSPIRATION REGARDING THE THREE LOCALITY \\ STATE OF ESPIRITO SANTO DURING THE DRY PERIOD
}

\author{
Edvaldo Fialho dos Reis ${ }^{1}$; Rosembergue Bragança ${ }^{2}$; Giovanni de Oliveira Garcia ${ }^{3}$; \\ José Eduardo Macedo Pezzopane ${ }^{4}$; Cristiano Tagliaferre ${ }^{5}$
}

\begin{abstract}
RESUMO
O presente trabalho teve como objetivo realizar o estudo comparativo das equações empíricas para estimativa da evapotranspiração de referência em comparação ao método universal padrão Penman-Monteith FAO 56 para as condições climáticas dos municípios de Sooretama, Cachoeiro de Itapemirim, Venda Nova do Imigrante localizados, respectivamentes, nas regiões Norte, Sul e Serrana do Estado do Espírito Santo. Foram utilizadas as variáveis climáticas referentes ao período seco (junho, julho e agosto) do ano de 2006 obtidas via plataforma de dados do CPTEC - INPE provenientes das estações automatizadas agrometeorologicas códigos $\mathrm{n}^{\circ} 31957,31958$ e 31959 instaladas, respectivamente, nos municípios mencionados. Para comparar os valores de ETo estimados por meio das equações empíricas com os do método universal padrão Penman-Monteith (FAO56) foram considerados os parâmetros da equação de regressão $\beta_{0}$ e $\beta_{1}$, coeficiente de determinação $\left(\mathrm{r}^{2}\right)$, coeficiente de correlação (r), estimativa do erro padrão (EEP), índice de concordância (d), índice de confiança ou desempenho (c), nas escalas diária, três, cinco e sete dias, para os meses estudados nas três localidades. Para as condições climáticas de onde se realizou o trabalho, em função dos índices e parâmetros estudados, para o período seco os melhores métodos pela ordem foram: Penman 48 Original, Priestley-Taylor (PT), FAO24 Penman Modificado, FAO24 Blaney-Criddle e Turc (61), FAO24 Radiação, Makkink (Mk).
\end{abstract}

Palavras-chave: Variáveis climáticas, equações empíricas, evapotranspiração de referência

\section{ABSTRACT}

This work had the objective of a comparative study of the empirical equations for the estimate of the reference evapotranspiration in comparison to Penman-Monteith FAO 56 universal standard method for the weather conditions from the municipalities of Sooretama, Cachoeiro de Itapemirim and Venda Nova do Imigrante located, respectively, in the north, south and mountainous regions of the Espirito Santo state. Weather variables regarding the dry period (June, July and August) in 2006 were used. They were obtained from data platform of CPTEC-INPE from the agrometeorological automated stations code number 31957, 31958 and 31959 installed respectively, in the municipalities showed. To make a comparison between the values of ETo estimated by the empirical equation and the universal standard method Penman-Monteith (FAO 56) there were considered the regression equation parameters $\beta_{0}$ and $\beta_{1}$, determination coefficient $\left(r^{2}\right)$, correlation coefficient $(r)$, standard of the error estimate (EEP), the agreement index $(d)$, confidence or performance index $(c)$ in the diary scales, three, five and seven days, for the months studied in the three

1 Departamento de Engenharia Rural, Centro de Ciências Agrárias da Universidade Federal do Espírito Santo, Alegre, ES, edreis@cca.ufes.br.

2 Departamento de Engenharia Rural, Centro de Ciências Agrárias da Universidade Federal do Espírito Santo, Alegre, ES,bragança@ cca.ufes.br

3 Departamento de Engenharia Rural, Centro de Ciências Agrárias da Universidade Federal do Espírito Santo, Alegre, ES, giovanni@cca.ufes.br

4 Departamento de Engenharia Florestal, Centro de Ciências Agrárias da Universidade Federal do Espírito Santo, Alegre, ES, jmep@cca.ufes.br

5 Departamento de Engenharia Agrícola, Universidade Federal do Sudoeste da Bahia, Viçosa, MG, tagliaferre@ yahoo.com.br

Fecha de Recepción: 11 Mayo 2007

Fecha de Aceptación: 02 Julio 2007 
localities. For the weather conditions where the work was done, according to the dry and rainy periods, the best methods were: Penman 48 Original, Priestley-Taylor (PT), FAO24 Penman Modificado, FAO24 Blaney-Criddle, Turc (61), FAO24 Radiação, Makkink (Mk) .

Key words: Weather variables, empiric equations, reference evapotranspiration.

\section{INTRODUÇÃO}

A atividade que mais consome água é a agricultura, com previsões futuras de aumentar esse consumo em face de demanda de alimentos e de uma população cada vez mais crescente. No Brasil, a partir da década de 80 ocorreu a maior expansão da Agricultura Irrigada, mais tecnificada, principalmente com adoção de novos métodos ou técnicas de Irrigação pressurizadas, estimando 1.515.379 hectares irrigados com aspersão, pivô central, localizada. A evolução da agricultura irrigada no estado do Espírito Santo se deu a partir de 1986 com o programa nacional de irrigação-PRONI, estimando uma área irrigada de 160.000 hectares, com aspersão convencional, autopropelido, microaspersão, gotejo e pivô central, só nas bacias: Itaunas, São Mateus e Doce Suruaca, têm-se aproximadamente 329 pivôs centrais, irrigando aproximadamente 20.000 ha. (Coelho, et al., 2004).

A necessidade de se produzir com qualidade, produtividade e maior quantidade de produtos alimentícios diversificados exige da comunidade científica novos conhecimentos sobre as reais necessidades hídricas das culturas, de determinada região, associados com sistemas ou métodos de irrigação, visando aplicar a quantidade certa e no momento certo que a planta requer, com adoção de manejo da água na propriedade, objetivando primeiramente a diminuição dos impactos ambientais negativos sobre o recurso natural água, no processo de irrigação, proporcionando o uso eficiente da água, com a utilização de métodos ou técnicas mais apropriadas, com o conhecimento dos estudos das variáveis climáticas que quantificam as necessidades hídricas das plantas.

O conhecimento das variáveis climáticas ou elementos meteorológicos registrados nas estações convencionais ou automáticas de agrometeorologia permite a quantificação da evapotranspiração das culturas, possibilitando assim conhecer os potenciais hídricos cultural diários, mensais e anuais da região, necessário para satisfazer as reais necessidades hídricas das culturas ali estabelecidas ou a serem implantadas.
Aproximadamente 8\% da área do estado apresentam déficit hídrico anual superior a $400 \mathrm{~mm}$, e $60 \%$ entre $200 \mathrm{~mm}$ a $400 \mathrm{~mm}$, evidenciando a grande limitação de alternativas de uso agrícolas dessas áreas nas condições naturais, necessitando do uso de irrigação sob ótica da produtividade e da redução dos riscos climáticos. Essa deficiência hídrica representa a fração da evapotranspiração potencial que não foi utilizada por limitação de disponibilidade hídrica (Ares, 2006).

Para o Estado do Espírito Santo, a quantificação da evapotranspiração assume particular importância em virtude dos déficits hídricos ao longo do ano constituírem uma limitação à produção agrícola e uma permanente fonte de risco agrícola, principalmente em áreas significativas, cujas características climáticas se aproximam da semi-aridez (Sacardua et al., 1986).

Diante do exposto, o presente trabalho teve como objetivo avaliar o desempenho dos métodos empíricos Penman (48) original, Penmam modificado (FAO24), Radiação (FAO24), Blaney-Criddle (FAO24), Hargreaves-Samani (1985), PriestleyTaylor (PT), Makkink (Mk) e Turc (1961) com o método universal proposto pela FAO como padrão Penman-Monteith (PM-FAO56) para três localidades do Estado do Espírito Santo.

\section{MATERIAIS E MÉTODOS}

O Trabalho foi conduzido espacializado nos municípios de Cachoeiro de Itapemirim (Latitude $20^{\circ}$ $42^{\prime}$ S, Longitude $41^{\circ} 17^{\prime} \mathrm{W}$, Altitude de $146,0 \mathrm{~m}$ ), Sooretama (Latitude de $19^{\circ} 22^{\prime} \mathrm{S}$, Longitude de $40^{\circ}$ 04' W, Altitude de 75,0 m) e Venda Nova do Imigrante (Latitude de $20^{\circ} 23^{\prime} \mathrm{S}$, Longitude de $41^{\circ}$ $11^{\prime} \mathrm{W}$, Altitude de 727,0 m) situados, respectivamente, nas regiões Norte, Sul e Serrana do Estado do Espírito Santo.

Para a estimativa da ETo foram coletadas em cada via plataforma de coletas de dados do CEPTEC/ INPE, as variáveis climáticas: pressão atmosférica, temperatura média, máxima e mínima do ar, umidade relativa média do ar, precipitação, radiação 
solar acumulada e velocidade do vento referentes ao período seco de 2006 (junho, julho e agosto) oriundas das estações automatizadas agrometeorológicas denominadas AGROMET instaladas nos municípios de Sooretama, Cachoeiro de Itapemirim e Venda Nova do Imigrante de códigos no 31957 , 31958 e 31959 , respectivamente.

Foi utilizado o Programa Computacional REF-ET, para a estimativa da ETo pelos métodos empíricos de Penman (48) original, Penman Modificado (FAO24), Radiação (FAO24), BlaneyCriddle (FAO24), Hargreaves-Samanni (85), Priestley-Taylor (PT), Makkink (Mk) e Turc (61) os quais foram comparadas com o método universal padrão Penman-Monteith (PM-FAO56) nas escalas diária, três, cinco e sete dias para períodos seco nas localidades descritas anteriormente.

Antes de se iniciar o trabalho, com o propósito de tornar os dados das variáveis agrometeorológicas mais homogêneos, foi feita uma verificação e posteriormente eliminação daquelas informações consideradas discrepantes, incompletas e até inconsistentes visando obter agrupamentos de dados mais representativos. Para fins de discussão, foi apresentado os resultados dos períodos seco (Junho, julho e agosto de 2006) das localidades estudadas.

Após a verificação dos dados foi realizada uma análise de regressão que correlacionou os valores de ETo estimados pelas equações empíricas com o método universal padrão FAO 56 PenmanMonteith (FAO56-PM). Foram considerados os parâmetros da equação de regressão $\beta_{0}$ e $\beta_{1}$, coeficiente de determinação $\left(\mathrm{r}^{2}\right)$, coeficiente de correlação (r).

A análise de desempenho dos modelos foi feita comparando os valores de ETo obtidos pelos métodos empíricos com o método universal padrão FAO 56 - Penman-Monteith (FAO56PM). A metodologia utilizada para comparar os resultados foi à estimativa do erro padrão (EEP) proposta por Allen et al. (1986) calculada por meio da equação:

$\mathrm{EEP}=\left(\frac{\sum(\mathrm{y}-\hat{\mathrm{y}})^{2}}{\mathrm{n}-1}\right)^{\frac{1}{2}}$

em que,

$\mathrm{EEP}=$ estimativa do erro padrão, $\mathrm{mm} \cdot \mathrm{dia}^{-1}$;

$\mathrm{y}=$ evapotranspiração de referência estimada pelo pelo método padrão, $\mathrm{mm}$. dia ${ }^{-1}$

$\hat{y}=$ evapotranspiração de referência obtida pelo considerado, mm. dia ${ }^{-1}$; e

$\mathrm{n} \quad=$ número de observações.

A precisão foi dada pelo coeficiente de determinação, o qual indica o grau em que a regressão explica a soma do quadrado total. A aproximação dos valores de ETo estimados por determinado método estudado, em comparação aos valores obtidos pelo método padrão, foi obtido pelo índice de concordância (d) (Willmott et al., 1985), onde seus valores variam de zero (não existe concordância) a "um" (concordancia perfeita). O valor de (d) é calculado por meio da seguinte equação:

$d=1-\frac{\sum_{i=1}^{n}(P i-O i)^{2}}{\sum_{i=1}^{n}[(|P i-\bar{O}|)+(O i-\bar{O})]^{2}}$

em que,

$\mathrm{d}$ = índice de concordância ou ajuste;

$\mathrm{Pi}=$ evapotranspiração de referência obtida pelo método considerado, mm.dia ${ }^{-1}$,

Oi = evapotranspiração de referência obtida pelo método padrão, mm.dia ${ }^{-1}$;

$\mathrm{O}=$ média dos valores de ETo obtida pelo método padrão' mm.dia ${ }^{-1}$; e

$\mathrm{n}=$ número de observações.

\section{RESULTADOS E DISCUSSÃO}

Segundo Thorntwaite (1948) a localidade de Sooretama apresenta a seguinte classificação climática: C2A'ra' Clima Úmido Subúmido (C2), Megatérmico (A'), pequena ou nenhuma deficiência hídrica (r), concentração de $33,3 \%$ da evapotranspiração potencial anual no trimestre mais quente (dezembro, janeiro e fevereiro). Por sua vez a localidade de Venda Nova do imigrante apresenta B3B'3 ra' clima úmido (B3), mesotérmico (B'3), pequena ou nenhuma deficiência hídrica (r), concentração de 33,2\% da evapotranspiração potencial anual no trimestre mais quente (dezembro, janeiro e fevereiro) e a localidade de Cachoeiro do Itapemirim apresenta C1A'da clima seco e Sub úmido (C1), megatérmico (A’), peque- 
Tabela 1

Parâmetros da equação de regressão $(\beta 0, \beta 1)$ coeficiente de determinação $\left(r^{2}\right)$, coeficiente de correlação(r), estimativa do erro padrão (EEP), índice de concordância (d), índice de confiança ou desempenho (c) e valores da ETo diárias, três, cinco e sete dias, para o período seco (junho, julho e agosto) de 2006 na localidade de Venda Nova do Imigrante-ES.

\begin{tabular}{|c|c|c|c|c|c|c|c|c|c|}
\hline Métodos & $\beta 0$ & $\beta 1$ & $\mathbf{r}^{2}$ & EEP & $\mathbf{r}$ & d & c & Classificação* & ETo (mm) \\
\hline \multicolumn{10}{|c|}{ Diária } \\
\hline Fao 56 PM & & & & & & & & & 2,36 \\
\hline Penman 48 & $-0,516$ & 1,154 & 0,865 & 0,385 & 0,93 & 0,840 & 0,78 & Bom & 2,20 \\
\hline $\begin{array}{l}\text { Fao } 24 \text { Pen. } \\
\text { mod }\end{array}$ & $-1,134$ & 1,410 & 0,870 & 0,531 & 0,93 & 0,891 & 0,83 & Muito bom & 2,19 \\
\hline Fao $24 \mathrm{Rd}$ & $-1,037$ & 1,636 & 0,887 & 0,754 & 0,94 & 0,803 & 0,76 & Bom & 2,82 \\
\hline Fao $24 \mathrm{BC}$ & 0,030 & 1,067 & 0,847 & 0,364 & 0,92 & 0,845 & 0,77 & Bom & 2,55 \\
\hline Harg-samani & 2,762 & 0,133 & 0,142 & 0,921 & 0,37 & 0,623 & 0,23 & Péssimo & 3,07 \\
\hline Priestley-taylor & 0,440 & 0,890 & 0,825 & 0,345 & 0,90 & 0,837 & 0,76 & Bom & 2,54 \\
\hline Makkink-57 & $-0,278$ & 1,039 & 0,778 & 0,432 & 0,88 & 0,864 & 0,76 & Bom & 2,17 \\
\hline Turc-61 & 0,295 & 0,995 & 0,668 & 0,537 & 0,81 & 0,821 & 0,67 & Mediano & 2,64 \\
\hline \multicolumn{10}{|c|}{ Três dias } \\
\hline Fao 56 PM & & & & & & & & & 2,33 \\
\hline Penman 48 & 0,013 & 0,928 & 0,930 & 0,225 & 0,96 & 0,965 & 0,93 & Ótimo & 2,18 \\
\hline $\begin{array}{l}\text { Fao } 24 \text { pen } \\
\text { mod }\end{array}$ & $-0,286$ & 1,050 & 0,894 & 0,280 & 0,94 & 0,954 & 0,90 & Mbom & 2,17 \\
\hline Fao $24 \mathrm{Rd}$ & $-0,438$ & 1,383 & 0,860 & 0,626 & 0,92 & 0,848 & 0,98 & Ótimo & 2,79 \\
\hline Fao 24 BC & 0,019 & 1,069 & 0,896 & 0,295 & 0,94 & 0,950 & 0,90 & Mbom & 2,51 \\
\hline Harg-samani & 1,030 & 0,861 & 0,790 & 0,775 & 0,88 & 0,717 & 0,63 & Mediano & 3,04 \\
\hline Priestley-taylor & 0,347 & 0,927 & 0,921 & 0,252 & 0,96 & 0,958 & 0,91 & Ótimo & 2,51 \\
\hline Makkink-57 & 0,024 & 0,910 & 0,799 & 0,344 & 0,89 & 0,924 & 0,82 & Mbom & 2,15 \\
\hline Turc-61 & 0,424 & 0,937 & 0,801 & 0,407 & 0,89 & 0,899 & 0,80 & bom & 2,61 \\
\hline \multicolumn{10}{|c|}{ Cinco dias } \\
\hline Fao 56 PM & & & & & & & & & 2,31 \\
\hline Penman 48 & 0,170 & 0,862 & 0,968 & 0,188 & 0,92 & 0,965 & 0,89 & Mbom & 2,17 \\
\hline $\begin{array}{l}\text { Fao } 24 \text { pen } \\
\text { mod }\end{array}$ & $-0,145$ & 0,991 & 0,949 & 0,208 & 0,97 & 0,963 & 0,93 & Ótimo & 2,15 \\
\hline Fao $24 \mathrm{Rd}$ & $-0,280$ & 1,323 & 0,852 & 0,590 & 0,92 & 0,820 & 0,75 & Bom & 2,78 \\
\hline Fao $24 \mathrm{BC}$ & 0,058 & 1,059 & 0,909 & 0,271 & 0,95 & 0,943 & 0,90 & Mbom & 2,51 \\
\hline Harg-samani & 0,813 & 0,956 & 0,812 & 0,774 & 0,90 & 0,676 & 0,60 & Mediano & 3,03 \\
\hline Priestley-taylor & 0,412 & 0,903 & 0,917 & 0,247 & 0,95 & 0,945 & 0,90 & Mbom & 2,50 \\
\hline Makkink-57 & 0,153 & 0,860 & 0,781 & 0,308 & 0,88 & 0,913 & 0,80 & Bom & 2,15 \\
\hline Turc-61 & 0,601 & 0,868 & 0,787 & 0,396 & 0,88 & 0,871 & 0,77 & Bom & 2,61 \\
\hline \multicolumn{10}{|c|}{ Sete dias } \\
\hline Fao 56 PM & & & & & & & & & 2,35 \\
\hline Penman 48 & 0,123 & 0,882 & 0,945 & 0,203 & 0,97 & 0,997 & 0,97 & Ótimo & 2,20 \\
\hline Fao24 pen mod & $-0,153$ & 0,994 & 0,906 & 0,239 & 0,95 & 0,995 & 0,95 & Ótimo & 2,18 \\
\hline Fao $24 \mathrm{Rd}$ & $-0,106$ & 1,239 & 0,788 & 0,592 & 0,88 & 0,980 & 0,87 & Mbom & 2,81 \\
\hline Fao 24 BC & 0,141 & 1,017 & 0,892 & 0,263 & 0,94 & 0,995 & 0,94 & Ótimo & 2,53 \\
\hline Harg-Samani & 0,878 & 0,925 & 0,854 & 0,759 & 0,92 & 0,970 & 0,89 & Mbom & 3,06 \\
\hline Priestley-taylor & 0,489 & 0,865 & 0,88 & 0,249 & 0,93 & 0,996 & 0,93 & Ótimo & 2,52 \\
\hline Makkink-57 & 0,309 & 0,786 & 0,696 & 0,351 & 0,83 & 0,991 & 0,82 & Mbom & 2,16 \\
\hline Turc-61 & 0,756 & 0,794 & 0,692 & 0,408 & 0,83 & 0,989 & 0,82 & Mbom & 2,62 \\
\hline
\end{tabular}

* Camargo \& Sentelha (1997). 
no ou nenhum excesso hídrico (d), concentração de $34,6 \%$ da evapotranspiração potencial anual no trimestre mais quente (dezembro, janeiro e fevereiro).

Na Tabela 1 encontram-se os parâmetros da equação de regressão $\left(\beta_{0}, \beta_{1}\right)$ coeficiente de determinação $\left(r^{2}\right)$, coeficiente de correlação (r), estimativa do erro padrão (EEP), índice de concordância (d), índice de confiança ou desempenho (c) e valores da ETo diária, três, cinco e sete dias, para o período seco (junho, julho e agosto) de 2006 para a localidade de Venda Nova do Imigrante-ES.

Os resultados obtidos no período seco de 2006 da localidade de Venda nova do Imigrante, na escala diária, mostram que o método de FAO 24 Penman Modificado apresentou o melhor índice de desempenho $(\mathrm{c}=0,83)$ caracterizado como Muito bom, com desempenho Bom ficaram os métodos de Penman 48, FAO 24 Radiação, FAO 24 Blaney-Criddle, Priestly-Taylor e Makkink e desempenho Mediano o de Turc e com índice de precisão $r(0,37)$ e c $(0,23)$ caracterizado como desempenho Péssimo o de Hargreaves-Samani, com elevado valor de $\operatorname{EEP}\left(0,921 \mathrm{~mm} \mathrm{dia}^{-1}\right)$ superestimando em $0,71 \mathrm{~mm} \mathrm{dia}^{-1}(30 \%)$ a ETo em relação ao padrão FAO 56 PM, portanto, com restrição de uso para as condições climáticas do local do trabalho, com restrição também para FAO 24 Radiação (EEP=0,754 $\mathrm{mm} \mathrm{dia}^{-1}$ ) e superestimando em $20 \%$ a ETo em relação ao padrão FAO 56 PM. Resultados semelhantes citados por Tagliaferre (2006), para as condições climáticas de Viçosa-MG.

$\mathrm{Na}$ análise dos resultados obtidos na escala de três dias todas as equações estudadas apresentaram altos índices de precisão "r", de desempenho "c", índice de concordância "d" próximo de "um", caracterizando concordância quase perfeita. Portanto as equações poderão ser utilizadas na estimativa da ETo paras condições climáticas onde foi conduzido o trabalho ajustadas ao padrão PM FAO 56.

Com relação à EEP o maior valor foi para Hargreaves-Samani $\left(0,775 \mathrm{~mm} \mathrm{dia}^{-1}\right)$ seguido pelo método de Radiação FAO $24\left(0,626 \mathrm{~mm} \mathrm{dia}^{-1}\right)$, superestimando a ETo em $0,71 \mathrm{~mm} \mathrm{dia}^{-1}(30 \%) \mathrm{e}$ o,46mm dia-1 ${ }^{-1}(20 \%)$ respectivamente, com restrição do uso dos mesmos para as condições climáticas do local de condução do trabalho. Resultados semelhantes na escala de três dias foram obtidos para as condições de Viçosa, MG, por Tagliaferre (2006).
$\mathrm{Na}$ análise dos resultados obtidos na escala de cinco dias todas as equações estudadas e comparadas com o padrão PM FAO-56, apresentaram altos índices de precisão "r" de desempenho "c", índice de concordância "d" próximo de "um", caracterizando concordância quase perfeita. Dessa forma as equações poderão ser utilizadas na estimativa da ETo ajustadas ao método padrão FAO56-PM para as condições climáticas da localidade do estudo.

Com relação à EEP o maior valor foi de Hargreaves-Samani $\left(0,774 \mathrm{~mm} \mathrm{dia}^{-1}\right)$, índice de desempenho "c" igual a 0,60 , superestimando a ETo em $0,72 \mathrm{~mm} \mathrm{dia}^{-1}(31 \%)$ em relação à ETo estimada pelo método padrão, portanto, não sendo recomendado seu uso para as condições climáticas do local da realização do trabalho. Restrição também ao método FAO 24 Radiação, pelo elevado valor de estimativa do erro padrão e superestimativa da ETo em $0,47 \mathrm{~mm} \mathrm{dia}^{-1}(20 \%)$. Resultados semelhantes foram obtidos por Tagliaferre (2006) para as condições climáticas de Viçosa-MG, onde os métodos Hargreaves-Samani superestimou a ETo em 33,7\% $\left(1,1 \mathrm{~mm} \mathrm{dia}{ }^{-1}\right)$ e FAO 24 Radiação superestimou a ETo em 10\% (0,33 $\left.\mathrm{mm} \mathrm{dia}^{-1}\right)$. Segundo o autor, o agrupamento das estimativas da ETo, tende a melhorar o desempenho dos parâmetros de quase todas equações.

Os resultados referente à escala de sete dias mostram que todos os métodos apresentaram alto índices de precisão "r", de desempenho "c", exatidão "d" próximo da unidade, caracterizando concordância quase perfeita, admitindo dessa forma a utilização das equações na estimativa da ETo ajustadas ao método padrão FAO 56-PM para as condições climáticas da localidade do estudo. Restrição apenas a método de ao Hargreaves-Samani em decorrência do elevado valor de $\operatorname{EEP}\left(0,759 \mathrm{~mm} \mathrm{dia}^{-1}\right)$, e conseqüentemente superestimativa da ETo em $0,71 \mathrm{~mm} \mathrm{dia}^{-1} \mathrm{em}$ relação ao obtido pelo método padrão. Resultado semelhante foi obtido por Tagliaferre (2006) para região de Viçosa, MG, na estimativa da ETo com uso do método de Hargreves-Samani, na escala de sete dias, obtendo alto valor da estimativa do erro padrão $\left(1,21 \mathrm{~mm} \mathrm{dia}^{-1}\right)$, superestimando a ETo em relação ao padrão FAO 56-PM.

Na Tabela 2 encontram-se os parâmetros da equação de regressão $\left(\beta_{0}, \beta_{1}\right)$ coeficiente de determinação $\left(r^{2}\right)$, coeficiente de correlação(r), estimativa do erro padrão (EEP), índice de concordância (d), índice de confiança ou desempenho (c) e valores da 
Tabela 2

Parâmetros da equação de regressão $\left(\beta_{0}, \beta_{1}\right)$ coeficiente de determinação $\left(\mathbf{r}^{2}\right)$, coeficiente de correlação (r), estimativa do erro padrão (EEP), índice de concordância (d), índice de confiança ou desempenho (c) e valores da ETo diárias, três, cinco e sete dias, para o período seco (junho, julho e agosto) de 2006 na localidade de Sooretama-ES

\begin{tabular}{|c|c|c|c|c|c|c|c|c|c|}
\hline Métodos & $\beta_{0}$ & $\beta_{1}$ & $\mathbf{r}^{2}$ & EEP & $\mathbf{r}$ & d & c & Classificação* & ETo $(\mathbf{m m})$ \\
\hline \multicolumn{10}{|c|}{ Diária } \\
\hline Fao $56 \mathrm{PM}$ & & & & & & & & & 2,48 \\
\hline Penman 48 & $-0,382$ & 1,106 & 0,842 & 0,370 & 0,91 & 0,736 & 0,67 & Mediano & 2,36 \\
\hline Fao 24 Pen mod & $-1,171$ & 1,399 & 0,853 & 0,534 & 0,92 & 0,716 & 0,66 & Mediano & 2,30 \\
\hline Fao $24 \mathrm{Rd}$ & $-0,749$ & 1,467 & 0,853 & 0,687 & 0,92 & 0,710 & 0,65 & Mediano & 2,89 \\
\hline Fao $24 \mathrm{BC}$ & 0,150 & 1,122 & 0,896 & 0,538 & 0,94 & 0,728 & 0,69 & Mediano & 2,93 \\
\hline Harg-samani & 2,231 & 0,413 & 0,385 & 0,961 & 0,62 & 0,559 & 0,34 & Mau & 3,26 \\
\hline Priestley-Taylor & 0,393 & 0,922 & 0,852 & 0,345 & 0,92 & 0,736 & 0,68 & Mediano & 2,68 \\
\hline Makkink-57 & $-0,110$ & 0,937 & 0,793 & 0,436 & 0,89 & 0,771 & 0,68 & Mediano & 2,21 \\
\hline Turc-61 & 0,367 & 1,000 & 0,734 & 0,567 & 0,85 & 0,722 & 0,61 & Mediano & 2,85 \\
\hline \multicolumn{10}{|c|}{ Três dias } \\
\hline Fao 56 PM & & & & & & & & & 2,47 \\
\hline Penman 48 & $-0,360$ & 1,099 & 0,932 & 0,233 & 0,96 & 0,971 & 0,93 & Ótimo & 2,36 \\
\hline Fao 24 Pen mod & $-1,078$ & 1,368 & 0,933 & 0,374 & 0,96 & 0,941 & 0,90 & Mbom & 2,30 \\
\hline Fao $24 \mathrm{Rd}$ & $-0,541$ & 1,384 & 0,875 & 0,589 & 0,93 & 0,871 & 0,81 & Mbom & 2,88 \\
\hline Fao $24 \mathrm{BC}$ & 0,256 & 1,074 & 0,896 & 0,510 & 0,94 & 0,876 & 0,83 & Mbom & 2,91 \\
\hline Harg-samani & 1,824 & 0,566 & 0,590 & 0,868 & 0,76 & 0,628 & 0,48 & Sofrível & 3,22 \\
\hline Priestley-Taylor & 0,326 & 0,946 & 0,907 & 0,279 & 0,95 & 0,953 & 0,90 & Mbom & 2,67 \\
\hline Makkink-57 & 0,007 & 0,887 & 0,823 & 0,387 & 0,90 & 0,907 & 0,82 & Mbom & 2,20 \\
\hline Turc-61 & 0,435 & 0,968 & 0,810 & 0,473 & 0,90 & 0,880 & 0,79 & Mbom & 2,83 \\
\hline \multicolumn{10}{|c|}{ Cinco dias } \\
\hline Fao 56 PM & & & & & & & & & 2,43 \\
\hline Penman 48 & $-0,212$ & 1,036 & 0,951 & 0,179 & 0,97 & 0,974 & 0,95 & Ótimo & 2,31 \\
\hline Fao 24 Pen mod & $-0,836$ & 1,266 & 0,955 & 0,282 & 0,97 & 0,949 & 0,92 & Ótimo & 2,25 \\
\hline Fao $24 \mathrm{Rd}$ & $-0,332$ & 1,303 & 0,875 & 0,521 & 0,93 & 0,852 & 0,79 & Bom & 2,84 \\
\hline Fao $24 \mathrm{BC}$ & 0,275 & 1,073 & 0,918 & 0,839 & 0,95 & 0,499 & 0,47 & Sofrível & 2,88 \\
\hline Harg-samani & 1,667 & 0,636 & 0,806 & 0,845 & 0,89 & 0,608 & 0,54 & Mediano & 3,21 \\
\hline Priestley-Taylor & 0,374 & 0,929 & 0,934 & 0,250 & 0,96 & 0,949 & 0,91 & Ótimo & 3,63 \\
\hline Makkink-57 & 0,167 & 0,826 & 0,832 & 0,342 & 0,91 & 0,894 & 0,81 & Mbom & 2,17 \\
\hline Turc-61 & 0,621 & 0,897 & 0,828 & 0,445 & 0,90 & 0,847 & 0,77 & Bom & 2,80 \\
\hline \multicolumn{10}{|c|}{ Sete dias } \\
\hline Fao 56 PM & & & & & & & & & 2,47 \\
\hline Penman 48 & $-0,370$ & 1,105 & 0,978 & 0,158 & 0,99 & 0,998 & 0,98 & Ótimo & 2,36 \\
\hline Fao24 Pen mod & $-0,994$ & 1,333 & 0,973 & 0,273 & 0,98 & 0,995 & 0,98 & Ótimo & 2,30 \\
\hline Fao $24 \mathrm{Rd}$ & $-0,130$ & 1,218 & 0,874 & 0,504 & 0,93 & 0,987 & 0,92 & Ótimo & 2,88 \\
\hline Fao 24 BC & 0,468 & 0,990 & 0,910 & 0,491 & 0,95 & 0,987 & 0,94 & Ótimo & 2,91 \\
\hline Harg-samani & 1,805 & 0,576 & 0,773 & 0,836 & 0,88 & 0,967 & 0,85 & Mbom & 3,23 \\
\hline Priestley-Taylor & 0,430 & 0,904 & 0,935 & 0,244 & 0,96 & 0,996 & 0,96 & Ótimo & 2,67 \\
\hline Makkink-57 & 0,313 & 0,764 & 0,824 & 0,357 & 0,90 & 0,991 & 0,90 & Mbom & 2,20 \\
\hline Turc-61 & 0,816 & 0,815 & 0,825 & 0,433 & 0,90 & 0,989 & 0,89 & Mbom & 2,83 \\
\hline
\end{tabular}

* Camargo \& Sentelha (1997). 
ETo diárias, três, cinco e sete dias, para o período seco (junho, julho e agosto) de 2006 para localidade de Sooretama/ES.

Os resultados obtidos no período seco (junho, julho e agosto de 2006) localidade de Sooretama, na escala diária todos os métodos estudados apresentaram desempenho Mediano, com índice médio de "c" $=0,66$, portanto novos estudos deverão ser realizados, por apresentarem resultados diferentes dos já obtidos no trabalho. Com relação ao método de Hargreaves-Samani ( $c=0,34, r=0,62 ; d=0,599$, $\left.\mathrm{EEP}=0,961 \mathrm{~mm} \mathrm{dia}^{-1}\right)$, caracterizando desempenho Mau, superestimando em 18\% a ETo em relação ao padrão FAO 56 PM, portanto com restrição de uso para estimativa da ETo, para as condições climáticas do local da pesquisa.

Os resultados obtidos na escala de três dias o melhor desempenho foi para o método de Penman (48) original que apresentou os seguintes indicadores $\mathrm{r}=0,96 ; \mathrm{d}=0,971 ; \mathrm{EEP}=0,233 \mathrm{~mm} \mathrm{dia}^{-1} \mathrm{e} \mathrm{c}=$ 0,93 classificando-se como ótimo e estimativa da ETo próximo do obtido pelo padrão, seguido pelos métodos FAO24 Penman Modificado $(\mathrm{c}=0,90)$, Priestley-Taylor $(\mathrm{c}=0,90)$, Makkink $(\mathrm{c}=0,79)$, FAO24 Blaney-Criddle $(c=0,83)$, portanto podem ser usados na estimativa da ETo para as condições climática do local, ajustados ao padrão FAO 56 PM. Restrição ao método de Hargreaves-Samani, com desempenho Sofrível, elevado EEP $=0,868 \mathrm{~mm}$ dia $^{-1}$, superestimando em $30 \%$ a ETo em relação ao método padrão.

Os resultados obtidos na escala de cinco dias os métodos com desempenho ótimo foram os de Penman (48), FAO 24 Penman Modificado, Makkink com desempenho Muito bom e os de Turc, FAO 24 Radiação com desempenho classificado como Bom, podendo serem utilizados sem restrição de uso na estimativa da ETo ajustados ao método padrão FAO56-PM, para as condições climáticas do local do estudo. Restrição apenas aos métodos de FAO 24 Blaney-Criddle $(c=0,47 ; d=0,499 ; \mathrm{EEP}=0,839$ $\mathrm{mm} \mathrm{dia}{ }^{-1}$ superestimando a ETo em $18 \%$ ) e ao método de Hargreaves-Samani $(c=0,54, d=0,608$; $\mathrm{EEP}=0,836 \mathrm{~mm} \mathrm{dia}^{-1}$, superestimando a ETo em $32 \%$ ) em relação ao padrão FAO56-PM.

Os resultados obtidos na escala de sete dias, todos os métodos apresentaram desempenho variando de Muito bom a Ótimo, com redução dos valores de estimativa do erro padrão, altos índices de precisão, exatidão e desempenho, portanto sem restrição de uso dos mesmos na estimativa da ETo ajustados ao método padrão FAO56-PM, para as condições climáticas do local do estudo.

Sediyama (1983) estudando métodos para a estimativa da ETP para Viçosa-MG, comparado com o método evapotranspirômetro de Thornthwaite, indicou como melhores, em ordem, o Tanque Classe A, Penman, Thornthwaite, Blaney-Criddle, Hamon, Papadakis e Piché.

Semelhante aos resultados obtidos por este trabalho, Mandelli et al. (2005) compararam valores estimados com as equações empíricas de HargreavesSamani, Thornthwaite, Thornthwaite modificado, Camargo, Camargo modificado e Radiação, com método Penmam-Monteith utilizando o coeficiente de desempenho "c" proposto por Camargo \& Sentelhas (1997) e concluíram que os métodos de estimativa de ETo que empregam a radiação solar incidente apresentaram desempenho superiores aos que utilizam somente valores da temperatura do ar e que dentre os métodos que utilizam somente a temperatura do ar o de Hargreaves-Samani foi o que apresentou o melhor desempenho para o município de Bento Gonçalves, RS.

$\mathrm{Na}$ Tabela 3 encontram-se os parâmetros da equação de regressão $\left(\beta_{0}, \beta_{1}\right)$ coeficiente de determinação $\left(r^{2}\right)$, coeficiente de correlação(r), estimativa do erro padrão (EEP), índice de concordância (d), índice de confiança ou desempenho (c) e valores da ETo diária, três, cinco e sete dias, para o período seco(junho, julho e agosto) de 2006 para localidade da Fazenda Experimental Bananal do Norte- Cachoeiro de Itapemirim-ES.

A análise e discussão dos resultados obtidos no período seco (junho, julho e agosto de 2006) localidade Fazenda Experimental Bananal do Norte - Cachoeiro de Itapemirim-ES, na escala diária, o melhor método foi o de Priestley-Taylor $(\mathrm{r}=0,84$; $\mathrm{d}=0,850$ e $\mathrm{c}=0,71$ ) caracterizando desempenho Bom, subestimando em 4,6\% a ETo em relação ao método padrão PM FAO 56. Com relação aos métodos de Penman (48) Original e FAO 24 Penman Modificado ambos apresentaram desempenho Bom, porém, com elevados valores de EEP, necessitando de novos estudos. Com desempenho Mediano ficaram os métodos FAO 24 Radiação, Makkink e Turc com elevados valores de EEP, e com desempenho Mau o FAO24 Blaney-Criddle, portanto, necessários novos estudos. Quanto ao método de HargreavesSamani obteve desempenho Mediano (c = 0,54 e EEP $=0,880 \mathrm{~mm} \mathrm{dia}^{-1}$ ), superestimando em 0,74 $\mathrm{mm} \mathrm{dia}^{-1}(26,2 \%)$ a ETo em relação ao padrão FAO 
Tabela 3

Parâmetros da equação de regressão $\left(\beta_{0}, \beta_{1}\right)$ coeficiente de determinação $\left(\mathbf{r}^{2}\right)$, coeficiente de correlação(r), estimativa do erro padrão (EEP), índice de concordância (d), índice de confiança ou desempenho (c) e valores da ETo diárias, três, cinco e sete dias, para período seco (junho, julho e agosto) de 2006 para localidade da Fazenda Experimental Bananal do Norte-Cachoeiro de Itapemirim-ES

\begin{tabular}{|c|c|c|c|c|c|c|c|c|c|}
\hline Métodos & $\beta_{0}$ & $\beta_{1}$ & $\mathbf{r}^{2}$ & EEP & $\mathbf{r}$ & d & c & Classificação* & ETo $(\mathrm{mm})$ \\
\hline \multicolumn{10}{|c|}{ Diária } \\
\hline Fao 56 PM & & & & & & & & & 2,82 \\
\hline Penman 48 & $-0,417$ & 1,065 & 0,770 & 0,520 & 0,87 & 0,830 & 0,72 & Bom & 2,70 \\
\hline Fao 24 Pen mod & $-1,115$ & 1,332 & 0,770 & 0,660 & 0,87 & 0,820 & 0,71 & Bom & 2,64 \\
\hline Fao $24 \mathrm{Rd}$ & $-0,428$ & 1,257 & 0,680 & 0,770 & 0,82 & 0,830 & 0,68 & Mediano & 3,11 \\
\hline Fao $24 \mathrm{BC}$ & 0,977 & 0,860 & 0,260 & 1,310 & 0,50 & 0,630 & 0,32 & Mau & 3,40 \\
\hline Harg-Samani & 1,473 & 0,739 & 0,660 & 0,880 & 0,81 & 0,670 & 0,54 & Mediano & 3,56 \\
\hline Priestley-Taylor & 0,446 & 0,798 & 0,710 & 0,450 & 0,84 & 0,850 & 0,71 & Bom & 2,69 \\
\hline Makkink-57 & 0,208 & 0,770 & 0,550 & 0,730 & 0,74 & 0,770 & 0,57 & Mediano & 2,38 \\
\hline Turc-61 & 0,611 & 0,841 & 0,560 & 0,620 & 0,75 & 0,830 & 0,62 & Mediano & 2,98 \\
\hline \multicolumn{10}{|c|}{ Três dias } \\
\hline Fao 56 PM & & & & & & & & & 2,79 \\
\hline Penman 48 & $-0,266$ & 1,013 & 0,880 & 0,342 & 0,94 & 0,960 & 0,90 & Mbom & 2,56 \\
\hline Fao 24 Pen mod & $-0,884$ & 1,255 & 0,891 & 0,384 & 0,94 & 0,956 & 0,90 & Mbom & 2,61 \\
\hline Fao $24 \mathrm{Rd}$ & $-0,114$ & 1,145 & 0,708 & 0,585 & 0,84 & 0,906 & 0,76 & Bom & 3,07 \\
\hline Fao $24 \mathrm{BC}$ & 0,706 & 0,950 & 0,471 & 0,893 & 0,68 & 0,808 & 0,55 & Mediano & 3,36 \\
\hline Harg-Samani & 0,715 & 1,002 & 0,613 & 0,803 & 0,78 & 0,835 & 0,65 & Mediano & 3,51 \\
\hline Priestley-Taylor & 0,417 & 0,806 & 0,799 & 0,327 & 0,89 & 0,958 & 0,85 & Mbom & 2,66 \\
\hline Makkink-57 & 0,307 & 0,738 & 0,793 & 0,640 & 0,89 & 0,850 & 0,75 & Bom & 2,36 \\
\hline Turc-61 & 0,741 & 0,790 & 0,652 & 0,444 & 0,80 & 0,929 & 0,75 & Bom & 2,95 \\
\hline \multicolumn{10}{|c|}{ Cinco dias } \\
\hline Fao 56 PM & & & & & & & & & 2,78 \\
\hline Penman 48 & $-0,175$ & 0,978 & 0,932 & 0,297 & 0,96 & 0,951 & 0,92 & Ótimo & 2,55 \\
\hline Fao 24 Pen mod & $-0,777$ & 1,214 & 0,942 & 0,305 & 0,97 & 0,957 & 0,93 & Ótimo & 2,60 \\
\hline Fao $24 \mathrm{Rd}$ & 0,118 & 1,066 & 0,754 & 0,509 & 0,86 & 0,880 & 0,76 & Bom & 3,08 \\
\hline Fao 24 BC & 1,166 & 0,791 & 0,540 & 0,782 & 0,73 & 0,739 & 0,54 & Mediano & 3,36 \\
\hline Harg-Samani & 0,742 & 0,993 & 0,900 & 0,777 & 0,94 & 0,752 & 0,71 & Bom & 3,50 \\
\hline Priestley-Taylor & 0,526 & 0,769 & 0,845 & 0,288 & 0,91 & 0,941 & 0,86 & Mbom & 2,66 \\
\hline Makkink-57 & 0,436 & 0,687 & 0,710 & 0,568 & 0,84 & 0,806 & 0,68 & Mediano & 2,35 \\
\hline Turc-61 & 0,944 & 0,722 & 0,684 & 0,410 & 0,82 & 0,885 & 0,73 & Bom & 2,95 \\
\hline \multicolumn{10}{|c|}{ Sete dias } \\
\hline Fao 56 PM & & & & & & & & & 2,79 \\
\hline Penman 48 & $-0,431$ & 1,072 & 0,988 & 0,255 & 0,99 & 0,996 & 0,99 & Ótimo & 2,57 \\
\hline Fao 24 Pen mod & $-1,039$ & 1,309 & 0,987 & 0,285 & 0,99 & 0,996 & 0,99 & Ótimo & 2,62 \\
\hline Fao $24 \mathrm{Rd}$ & $-0,007$ & 1,106 & 0,759 & 0,507 & 0,87 & 0,989 & 0,86 & Mbom & 3,09 \\
\hline Fao $24 \mathrm{BC}$ & 0,765 & 0,926 & 0,715 & 0,695 & 0,84 & 0,981 & 0,82 & Mbom & 3,35 \\
\hline Harg-Samani & 0,968 & 0,916 & 0,905 & 0,789 & 0,95 & 0,976 & 0,93 & Ótimo & 3,53 \\
\hline Priestley-Taylor & 0,411 & 0,808 & 0,890 & 0,256 & 0,94 & 0,996 & 0,94 & Ótimo & 2,67 \\
\hline Makkink-57 & 0,380 & 0,708 & 0,742 & 0,559 & 0,86 & 0,982 & 0,84 & Mbom & 2,36 \\
\hline Turc-61 & 0,836 & 0,756 & 0,682 & 0,402 & 0,82 & 0,992 & 0,82 & Mbom & 2,95 \\
\hline
\end{tabular}

* Camargo \& Sentelhas (1997). 
56 PM, portanto com restrição de seu uso para as condições climáticas do local do estudo.

Os resultados na escala de três dias, os melhores métodos foram Penman 48, FAO 24 Penman Modificado, Priestley-Taylor com desempenho Muito bom, valores da estimativa do erro padrão relativamente baixos, com altos índices de precisão e exatidão, portanto sem restrição do uso dos mesmos na estimativa da ETo, ajustados ao padrão FAO56-PM, por apresentarem subestimativas de ETo.

Quanto aos métodos FAO24 Radiação, Makkink e Turc, apresentaram desempenho Bom, porém com elevados valores de EEP, necessitando de novos estudos. Com desempenho Mediano os métodos

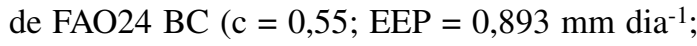
$\mathrm{r}=0,68)$ e Hargreaves-Samani (HS) $(\mathrm{c}=0,65$; $\left.\mathrm{EEP}=0,803 \mathrm{~mm} \mathrm{dia}^{-1} ; \mathrm{r}=0,78\right)$, superestimando a ETo em $20,4 \%$ e $25,8 \%$ respectivamentes em relação ao padrão FAO56 PM, portanto com restrição de uso dos mesmos para as condições climáticas locais sem novos estudos.

Os resultados obtidos na escala de cinco dias apresentaram como os melhores métodos Penman (48) e FAO24 Penman Modificado ambos com índice de desempenho igual a 0,90 - caracterizando desempenho Ótimo, altos índices de precisão e de exatidão e com valores relativamente pequenos do EEP, seguido pelo método de Priestley-Taylor $\left(c=0,86 ; \mathrm{r}=0,91 ; \mathrm{EEP}=0,288 \mathrm{~mm} \mathrm{dia}^{-1}\right)$, ambos com pequenas subestimativas de ETo em relação ao método padrão, portanto sem restrição de uso para a estimativa de ETo para as condições climáticas do local do estudo. Os métodos FAO 24 Radiação, Turc e Hargreaves-Samani apresentaram desempenho Bom, porém com elevados valores de EEP, necessitando de novos estudos. Já os métodos de Makkink $(c=0,68)$ e FAO24 Blaney-Criddle ficaram com desempenho Mediano e com valores elevados de EEP, portanto, com restrições ao uso.

Os resultados obtidos na escala de sete dias apresentaram melhores resultados os métodos de Penman (48) $\left(\mathrm{c}=0,99 ;\right.$ EEP $=0,297 \mathrm{~mm} \mathrm{dia}^{-1}$; $\mathrm{r}=0,96)$ e FAO 24 Penman Modificado $(\mathrm{c}=0,99$; $\mathrm{EEP}=0,305 \mathrm{~mm} \mathrm{dia}^{-1}$ e $\left.\mathrm{r}=0,97\right)$ e Priestley-Taylor ( $\left.c=0,94 ; \operatorname{EEP}=0,256 \mathrm{~mm} \mathrm{dia}^{-1} ; \mathrm{r}=0,94\right)$, com índices de concordância bem próximo da unidade, caracterizando concordância quase perfeita, com pequenas subestimativas da ETo em relação ao padrão FAO56-PM, portanto sem restrição de uso dos mesmos, para as condições climáticas do local do trabalho. Já os métodos de HargreavesSamani, FAO24 Blaney-Criddle, Turc e FAO 24 Radiação apresentaram desempenhos variando de Muito bom a Ótimo, porém com elevados valores de EEP, necessitando de novos estudos.

Segundo Smith (1991) a FAO recomenda que os métodos empíricos de estimativas da ETo, sejam calibrados e validados para outras regiões, sendo a equação de Penman-Monteith a referência padrão para esses ajustes.

Allen et al. (1998) citam que em estudos realizados no EUA e Europa, confirma-se a precisão e consistência do método de Penman-Monteith para clima úmido e seco.

Camargo \& Sentelha (1997) avaliaram o desempenho de vinte métodos de estimativas da ETo, com dados de três localidades de São Paulo, levando em consideração as medições lisimétricas para o período de 1954 à 1960. A avaliação dos dados foram baseados no coeficiente de correlação (r), de concordância (d) de Willmott e de um índice proposto de confiança ou desempenho (c) que é o produto (r.d). Os resultados obtidos demonstraram um desempenho muito bom $(0,78$ a 0,81$)$ para os métodos de Thornthwaite, Camargo et al. (1999) e Priestley-Taylor.

Alves Sobrinho et al., (1997) compararam a ETo estimada pelos métodos Penman-FAO, Hargreaves, Radiação-FAO, Blaney-Criddle e tanque classe A, com o método padrão PenmanMonteith, considerando períodos mensais, para duas localidades no estado de Mato Grosso do Sul, com exceção do método Tanque classe A-FAO, os resultados encontrados apresentaram altos níveis de correlação, indicando a possibilidade de se utilizar métodos empíricos que exigem poucas variáveis climatológicas na estimativa da ETo, baseados ou ajustados no método de Penman-Monteith.

\section{CONCLUSÕES}

- Para as condições climáticas registradas nos períodos seco de 2006 da localidade de Venda Nova do Imigrante, Sooretama e Cachoeiro de Itapemirim os métodos de estimativa de ETo para as quatro escalas temporais estudadas os melhores resultados foram obtidos por Penman (48) Original, FAO 24 Penman Modificado, Priestley-Taylor (PT), FAO24 Blaney-Criddle, Makkink (Mk), Turc, FAO24 Radiação, e; 
- Para as três localidades estudadas, destacaram-se como melhores métodos Penman (48) Original,
FAO 24 Penman Modificado, Priestley-Taylor (PT), FAO24 Blaney-Criddle e Turc (61).

\section{REFERÊNCIAS}

ALLEN, R.G.A 1986. Penman for all seasons. Journal of Irrigation and Drainage Engineering, New York, v. 112, n. 4 , p. 348-386.

ALLEN, R.G.; PEREIRA, L.S.; RAES, D.; SMITH, M. 1998. Evapotranpiração de colheitas - Diretrizes para computar necessidades de água de colheitas - irrigação e drenagem -Boletim FAO 56 - Roma.

ALVES SOBRINHO, T.; BONONO, R.; MANTOVANI, E.C.; SEDYAMA, G.C. 1997. Estimativa da evapotranspiração de referencia (ETo) para dourados e Ponta Porã, Mato Grosso do Sul. In: CONGRESSO BRASILEIRO DE ENGENHARIA AGRICOLA. 26, 1997, Campina Grande. Anais... campina Grande: SBEA, CD-Rom.

ATLAS DAS ÁREAS COM POTENCIAL DE RISCOS DO ESTADO DO ESPÍRITO SANTO, Vitória: GEES, 2006. 125 p.

CAMARGO, A.P. ; SENTELHAS, P. C. 1997. Avaliação do desempenho de diferentes métodos de estimativa da evapotranspiração potencial no estado de São Paulo. Revista Brasileira de Agrometeorologia, v. 5,n. 1. p. 89-97.

COELHO, R.D.; SCHMIDT W.; JACOMAZZI, M.A.; ANTUNES, M.A.H. 2004. Distribuição espacial de pivôs centrais no Brasil: I - região sudeste. Revista Brasileira de Engenharia Agrícola e Ambiental, Campina Grande, v. 8 , n. $2-3$, p. 7 .
MANDELLI, F.; CONCEIÇÃO, M.A.F. 2005. Comparação entre métodos de estimativa da evapotranspiração de referência em Bento Gonçalves, RS. Revista Brasileira Agrometeorologia, Santa Maria, v. 13, n. 2, p. 303-307.

SCARDUA, J.A.; FEITOZA, L.R.; CASTRO, L.L.F. 1986. Estimativas da evapotranspiração potencial para o Estado do Espírito Santo. $2^{\mathrm{a}}$ Ed. Vitória: Encapa, 44 p. (Boletim de pesquisa n. 6)

SEDIYAMA, G.C.; BERNARDO, S. et al. 1983. Estudo de métodos para estimativa da evapotranspiração potencial em Viçosa-MG. Experientiae, v. 16, n. 4, p. 74.

SMITH, M. 1991. Report on the expert consultation on revision of FAO methodologies for crop water requeriments. Rome: FAO, 45 p.

TAGLIAFERRE, C. 2006. Desempenho do irrigâmetro e de dois tipos de minievaporímetros para estimativa da evapotranspiração de referência. 110f. Tese (Doutorado)UFV, Viçosa.

THORNTHWAITE, C.W. 1948. An approach toward a rational classification of climate. The Geographical Review. New York, v. 38, n. 1, p. 55-94.

WILLMOTT, C.J.; CKLESON, S.G.; DAVIS, R.E. 1985. Statistics for evaluation and comparison of models. Journal of Geophysical Research, Ottawa, v. 90, n. C5. p. $8995-9005$. 